\section{Townsend's Ionization Coefficient for Helium}

AcCurate experimental data are lacking for Townsend's ionization coefficient $\alpha / p$ for electrons drifting through helium, when the ratio $X / p$ of electric field to pressure is small. As has been pointed out by Loeb ${ }^{1}$, however, it is possible in this instance to calculate $\alpha / p$ from the relation ${ }^{2}$

$$
\alpha / p=W^{-1} \int_{1}^{\infty} v f(V) P(V) d V,
$$

where $W$ is the drift velocity of the electrons, $v$ the speed of an electron which has energy $V$ in its random motion, $f(V)$ the energy distribution function in the random motion, $I$ the ionization energy, and $P(V)$ the probability of ionization for an electron with energy $V$ per unit random path at unit pressure, since the form of $f(V)$ has been determined with considerable accuracy by Smit $^{3}$.

\begin{tabular}{|c|c|c|c|c|c|c|}
\hline$X / p$ & 3 & 4 & 5 & 6 & $\frac{8}{10}$ & $\frac{10}{6}$ \\
\hline$\frac{17 \cdot 5}{\alpha / p}$ & $\frac{23 \cdot 5}{7 \times 10^{-5}}$ & $\frac{3 \cdot 0 \times 10^{-3}}{1 \cdot 0 \cdot 2 \times 10^{-3}}$ & $\frac{37 \cdot 5}{7 \cdot 7 \times 10^{-8}}$ & $\frac{52 \cdot 0}{2 \cdot 4 \times 10^{-2}}$ & $\frac{68 \cdot 0}{4 \cdot 6 \times 10^{-2}}$ \\
\hline
\end{tabular}

The table contains a number of values of $\alpha / p$ computed in this way", for values of $X / p$ between 3 and 10 volts $/ \mathrm{cm} . /(\mathrm{mm}$. mercury) together with the drift velocities $(W)$ employed, in $10^{5} \mathrm{~cm} . / \mathrm{sec}$. The greatest uncertainty probably occurs in the values of $W$. The values adopted are close to those calculated by $\mathrm{Smit}^{5}$, and are rather less than those given directly or by extrapolation from the experiments of Bradbury and Nielsen ${ }^{6}$. When the values of $\alpha / p$ are plotted on a logarithmic scale against $\log X / p$, a line is obtained which can be extrapolated to somewhat below that giving the values of $\alpha / p$ found experimentally by Townsend and McCallum? for values of $X / p$ greater than 12 .

\section{Queen's University,}

\section{S. H. DuNLOP} Belfast.

March 24.

'Loeb, "Fundamental Processes of Electrical Discharge in Gases", 365 (New York, 1939).

${ }^{2}$ Emeleus, Lunt, and Meek, C. A., Proc. Roy. Soc., A, 156, 394 (1936).

'Smit, Physica, 3, 543 (1936).

- Dunlop, M.Se. thesis, Belfast (1948).

s Druyvesteyn and Penning, Rev. Mod. Phys., 12, 97 (1940).

${ }^{6}$ Loeb, op. cit., 192.

Townsend and MacCallum. Phil. Mag., 17, 678 (1934).

\section{Coating of Microscope Eyepiece Lenses}

IN recent years the coating or 'blooming' of lenses has been widely practised and has proved a most effective means of eliminating undesirable reflexions. The process has been applied to the microscope, and the coating of objectives for use with normal incident illumination is quite common practice. It is not generally recognized, however, that in certain conditions the coating of eyepiece surfaces may be advantageous, and sweeping statements have appeared to the effect that no useful purpose is served by coating eyepiece lenses.

In any eyepiece multiple reflexion images are produced from the lens surfaces. Fig. 1 shows the

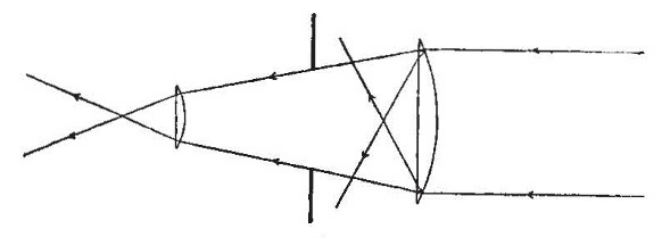

Fig. 1

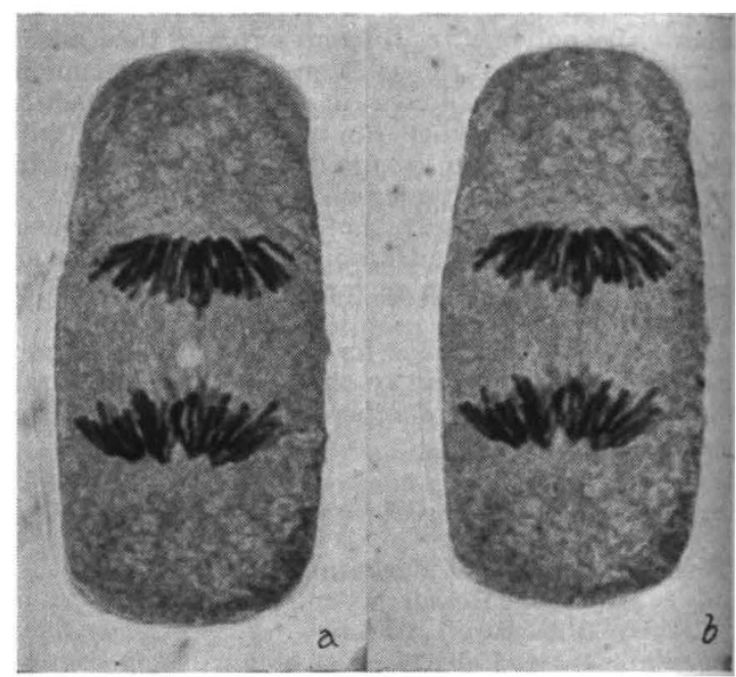

Fig 2

position of the first and brightest image produced by the field lens of a Huygenian eyepiece, and it will be seen that it is well behind the field stop. As this stop is at the principal focus of the eye lens, the image will therefore not be focused by the eye. In the widely used eyepiece, designed by Conrady, with variable separation between two corrected components (so that correction can be suited to different types of objective) the case is quite different. The reflexion image may at certain settings be brought into focus, and it appears in the centre of the field as a flare spot or a more or less sharp image of the light source. Visually this flare spot is not very noticeable unless the aperture of illumination is reduced; but it may be quite obvious when the eyepiece is used for photography. This is shown in Fig. $2 a$ (a photograph of mitosis in a cell of Libocedrus taken with $2-\mathrm{mm}$. oil-immersion apochromatic objective and variable separation $\times 14$ eyepiece); the flare spot appears in the centre of the photograph. It can, however, be quite easily eliminated by coating one or both surfaces of the field lens of the eyepiece. In practice, it is sufficient to coat one surface only, preferably the lower convex surface, as it is less likely to accumulate dust. It is important to insist on a good clean coating, as any flaws will appear as dark spots on the field. The so-called 'hard coating' is preferable as it is not so liable to damage. The photograph shown in Fig. $2 b$ was taken under the same conditions as that shown in Fig. $2 a$, but a field lens with one surface coated has been substituted for the untreated component; it will be seen that the flare spot has been eliminated.

G. R. LANE

Department of Botany,

University College,

Southampton.

March 23. 